\title{
Estudios sobre la relación familia-escuela (2008-2018): un análisis bibliométrico de la producción académica en español
}

\author{
Studies on the Family-School Relationship \\ (2008-2018). An Analysis of Academic \\ Production in Spanish
}

\section{Elsa María Castrillón-Correa ${ }^{1}$, Andrea Precht Gandarillas ${ }^{1}$, Jorge Valenzuela ${ }^{1}$ y Jean Nikola Cudina ${ }^{2}$}

${ }^{1}$ Universidad Católica del Maule, Chile

${ }^{2}$ Fundación Universitaria Católica Lumen Gentium - UNICATÓLICA, Colombia

\section{Resumen}

\begin{abstract}
Este artículo analiza la producción académica sobre la relación familia-escuela desde investigaciones en lengua española durante la década 2008-2018. Se analizaron 564 artículos indexados en las bases de datos Web of Science (WoS), Scopus y SciELO. Se utilizó el método bibliométrico descriptivo para analizar la producción académica y caracterizar las dinámicas de investigación de estos estudios, mediante un análisis de redes sociales académicas. Se utilizó el software VOSviewer 16.1 para diseñar mapas bibliométricos basados en texto y datos bibliográficos. Se constata que la producción académica no presenta una muestra clara de crecimiento, por el contrario, se reconocen fluctuaciones dadas por incrementos y disminuciones en las publicaciones. Se identifica la aparición de nichos de trabajo configurados por comunidades académicas conformadas en promedio por tres autores, sin evidencia de redes de colaboración entre ellas. El mayor impacto de la producción académica en términos de citación se encuentra en las bases de datos Scopus y WoS. El análisis de contenido de los doce artículos más citados muestra una alta heterogeneidad en torno a las temáticas desde las cuales se articulan los estudios de familia-escuela. Es razonable pensar que hace falta una mayor producción científica en español respecto de este campo, que consolide el abordaje de la relación familia-escuela.
\end{abstract}

Palabras clave: familia; escuela; relación familia-escuela; producción académica; comunidades académicas; educación; bibliometría

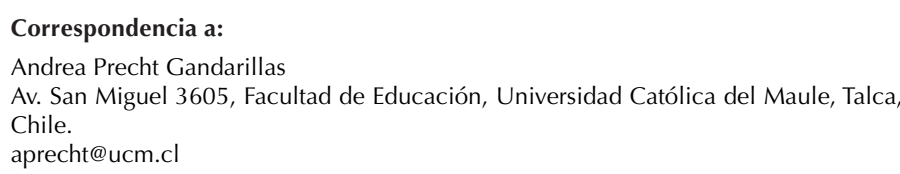

aprecht@ucm.cl

(C) 2021 PEL, http://www.pensamientoeducativo.org - http://www.pel.cl

ISSN:0719-0409ＤDI:203.262, Santiago, Chile doi: 10.7764/PEL.58.2.2021.12 


\title{
Abstract
}

\begin{abstract}
This paper aims to analyze academic production on the family-school relationship from research in Spanish during the last decade (2008-2018). A total of 564 papers indexed in the Scopus, Web of Science (WoS), and SciELO databases were analyzed. To do this, the descriptive bibliometric method was used to analyze the academic production and characterize the research dynamics of these studies, through an analysis of academic social networks. The VOSviewer 16.1 software was used to design bibliometric maps based on text and bibliographic data. The results show that academic production does not present clear growth; on the contrary, there are fluctuations due to increases and decreases in publications. Also observed are niches of work formed through small academic communities of a closed nature. The greatest impact of academic production in terms of citations is to be found in the Scopus and WoS databases. On the other hand, the content analysis of the 12 most cited papers shows high heterogeneity regarding the themes around which the family-school studies are articulated. Given these results, it is reasonable to consider that more scientific production in Spanish is needed in this field to provide evidence to consolidate the approach to and discussion of the family-school relationship..
\end{abstract}

Keyword: family; school; family-school relationship; academic production; academic communities; education; bibliometric

\section{Introducción}

En este estudio concebimos la relación familia-escuela como los vínculos existentes entre las personas pertenecientes a estas instituciones que se encuentran, socializan y se comunican entre sí, con el propósito de apoyar la trayectoria educativa escolar de un niño, niña o adolescente (en adelante, NNA). Diversos estudios reconocen la importancia de esta relación para el aprendizaje escolar (Jeynes, 2005, 2017; Tan, Lyu \& Pen, 2020).

Las aproximaciones para entender la relación entre familias y escuelas varían desde perspectivas que ponen el acento en la dimensión comunitaria (Ortega \& Cárcamo, 2018) o miradas más individualistas, apoyadas en el modelo de Epstein de alianza entre escuela y familia, cuya perspectiva está más centrada en las necesidades de las escuelas (Epstein, 2010; Baquedano-López, Alexander \& Hernández, 2013).

La forma en cómo se configura está relación variará según las condiciones materiales y el origen de las familias, pero la clase y la raza son factores importantes para las posibilidades de interacción de estas con las escuelas (Vincent, 2014). Asimismo, la ortodoxia neoliberal ha cambiado tanto los modos de concebir al otro en las escuelas (Riquelme, 2015) como la forma en la que se despliega la infancia (Peña, Ibarra \& Del Solar, 2014; Vergara-del Solar, Peña, Chávez \& Vergara 2015; Chávez \& Vergara, 2017), la parentalidad (Faircloth \& Rosen, 2020) y las relaciones filiales (Vergara-del Solar, Chávez-Ibarra, Peña-Ochoa \& Vergara-Leyton, 2016; Vergara-del Solar, Sepúlveda \& Salvo, 2019).

La investigación situada respecto de las relaciones entre familia-escuela se justifica tanto por la relevancia de esta relación para el aprendizaje escolar como por lo complejo de las relaciones de poder que la atraviesan y los cambios culturales que la impactan. En el caso de Hispanoamérica, se hace relevante conocer los estudios asociados a esta temática y, aunque los hay, a la fecha del sometimiento de este artículo y tras una detallada 
revisión, se pudo observar que no hay estudios bibliométricos que permitan analizar la producción académica en español para este campo en la última década. Por este motivo, el objetivo de este estudio fue analizar la producción científica en español que estudia la relación familia-escuela.

\section{Respecto de la necesidad de un estudio bibliométrico sobre la relación familia-escuela}

En la última década, la producción académica de las investigaciones sobre la relación familia-escuela parecieran tener un mayor crecimiento. Esto en parte por el alcance que han tenido los escenarios institucionales en que familias y escuelas se constituyen como objeto de estudio en el campo de la educación (Egido Gálvez, 2015; De León Sánchez, 2011; Bolívar, 2006). En el mundo hispanohablante, es posible encontrar un campo disciplinar que aborda la relación familia-escuela desde diferentes aristas, como las miradas desde las familias, docentes y apoderados, y otros actores escolares (Gubbins, 2014, 2016; Precht, 2016, 2018; Rodríguez-Triana, 2018; Cárcamo $\&$ Garreta, 2020), lo que permite dar cuenta de una comunidad académica activa. Algunos estudios previos se han centrado en indagar en qué grado se está estudiando la forma en cómo los padres educan a sus hijos (RuízMarín \& Hernández-Prados, 2016) o en describir la evolución de la participación de las familias en la escuela, cuando hay alumnos con necesidades específicas de apoyo educativo (Navarro, Sánchez \& Gómez, 2020).

Prevalecen investigaciones de corte meta-analítico que abordan los factores que afectan la participación de los padres en la educación escolar de sus hijos (Jafarov, 2015; Fan \& Chen, 2001). Otros estudios abordan el concepto de agencia en estudiantes y familias en la adaptación sociocultural a nuevos contextos escolares (por ejemplo, Castrillón-Correa, Cossio-Bolaños, Cudina, Gómez-Campos \& Precht, 2020) y el impacto de las relaciones familia-escuela en la inclusión educativa de alumnos (Carmona-Santiago, García, Máiquez \& Rodrigo, 2019), así como la relación familia-escuela en España (Gálvez, 2020).

El análisis de la producción académica de la relación entre familia-escuela se ha realizado de manera local; así, Castelli y Pepe (2008) realizaron un análisis bibliométrico-descriptivo de la producción académica anglosajona, registrada en este campo en la BBDD CSA (ex Cambridge Scientific Abstracts) de los años 1966 al 2005. Por su parte, Ávila y Moreno (2020) indagan sobre la forma en la que se aborda la relación familia-escuela en artículos académicos colombianos para el quinquenio 2012-2017.

Si bien estos estudios representan un antecedente importante, es necesario revisar la producción más reciente, pues estamos ante una escasez de investigaciones que analicen las dinámicas de investigación en este campo. Específicamente, cabe preguntarse por las características de la producción en español durante la última década, puesto que este idioma es el utilizado por la mayor parte de los actores educativos latinoamericanos, en desmedro del dominio de la lengua inglesa (http://www.ef.se/epi). A estos actores educativos les corresponde diseñar o implementar políticas específicas respecto de la relación familia-escuela. En esta perspectiva, los aportes en este campo están mediados por el acceso a la lengua, aun cuando algunos tomadores de decisión accedan a la producción anglófona.

En su análisis de la producción científica en español entorno a la relación entre familia-escuela, este estudio busca proveer información sobre las dinámicas de investigación de esta área en la región. Para ello, opta por emplear un método bibliométrico sobre los análisis sistemáticos meta-analíticos. Específicamente, cabe preguntarse por el estado y las características de esta producción en la última década, en especial respecto de: a) el impacto de la producción académica, b) las redes de coautoría que se establecen entre países e investigadores, c) las comunidades académicas que se conforman y d) dar a conocer de manera general las investigaciones más citadas que están abordando la relación familia-escuela. Consecuentemente, el objetivo de este estudio fue analizar la producción científica en español que aborda los estudios entorno a la relación entre familia-escuela. 


\section{Método}

Se lleva a cabo un análisis de la producción académica sobre la relación familia-escuela indexadas en las bases de datos (en adelante BBDD) de Scopus, WoS y Scielo en el periodo 2008-2018. Para ello, se utiliza el método bibliométrico, que permite comprender las dinámicas de investigación conformadas al interior del campo de conocimiento en el marco de un contexto social e histórico que hace posible su desarrollo (Mingers \& Leydesdorff, 2015; Millán, Polanco, Ossa, Béria \& Cudina, 2017). Esto posibilita evaluar el impacto de la investigación y revelar indicadores de producción, circulación, colaboración y citación. Estos dan a conocer las tendencias que se establecen al interior de la disciplina (Mingers \& Leydesdorff, 2015). Un indicador que tradicionalmente se usa para llevar a cabo la evaluación de la actividad científica se encuentra asociado a la productividad que emerge al interior de un área de conocimiento (Gómez-Morales; 2015; Tomás-Gorriz \& Tomás-Casterá, 2018). Si bien los métodos bibliométricos no garantizan -con exactitud y precisión- una imagen completa de la actividad investigadora, brindan recursos y técnicas para el análisis de la producción académica en grandes volúmenes de datos (Abramo \& D’Angelo, 2011).

Se expone un panorama de la producción académica en el campo de los estudios de la relación familia-escuela respecto de las publicaciones en idioma español, la principal lengua en Latinoamérica. Para reducir la brecha de la producción académica en español indexada en las BBDD de WoS y Scopus, hemos tomado la decisión de incluir SciELO, dado que esta BBDD incluye un mayor número de revistas indexadas por pares en América Latina, así como una producción académica importante en español. SciELO presenta indicadores bibliométricos que permiten comprender sus dinámicas de producción a nivel regional en esta parte del mundo. Además, se realiza un análisis de contenido a doce de los artículos más citados de la base de datos.

\section{Corpus}

El corpus empírico de este estudio corresponde a la producción académica en español de artículos de investigación que abordan la relación familia-escuela en las BBDD de WoS, Scopus y SciELO en las áreas de educación, ciencias sociales y psicología. Los heurísticos de búsqueda empleados para el rastreo del corpus empírico, en español e inglés, fueron los siguientes: ["relación" or "school-family relationship" or "school family relationship" or "familia-escuela" or "school-family"]. La mayoría de las revistas solicitan incorporar palabras clave en inglés, incluso si el artículo está en español; incluimos estas en la búsqueda para no perder información. La búsqueda se realizó el 10 de abril de 2019. Para determinar los artículos publicados en español tomamos como referencia las publicaciones declaradas e indexadas en español. Un total de 564 artículos constituye el corpus empírico del estudio y se distribuye de la siguiente manera: WoS ( $\mathrm{n}=88)$, Scopus ( $\mathrm{n}=277)$ y SciELO ( $\mathrm{n}=199)$.

\section{Procedimiento y análisis}

Una vez constituido el corpus empírico, se construyó la BBDD que incluye: autores, año de publicación, citaciones, países y revistas. Luego se procedió a analizar la producción académica. Para determinar indicadores de impacto y consumo se llevó a cabo una depuración manual de los datos. Se evidencian 28 revistas que comparten al menos la indexación con dos BBDD, por ello 43 artículos fueron excluidos. Los criterios de selección para depurar fueron dos: 1) artículos que reportan mayor número de citas de las BBDD; 2) para los artículos que presentaron el mismo indicador de citación o que no tienen registro de citación, se privilegió la indexación más alta, en el orden WoS, Scopus y SciELO. A partir de estos criterios de inclusión se obtuvo un total de 564 artículos indexados, que constituyen el corpus para el análisis de este estudio'. Se usaron técnicas bibliométricas

1. En el siguiente enlace el lector podrá tener acceso a la base de datos que constituyó el corpus empírico de este estudio: t.ly/5JAK 
(Cudina \& Ossa, 2016; Salas et al., 2018), específicamente el análisis de redes sociales académicas (Maltseva \& Batagelj, 2019) para mapear las comunidades académicas e identificar las redes de colaboración que se establecen entre investigadores y países al interior del campo.

Las redes de colaboración científica se constituyen en objeto de análisis para develar las comunidades académicas que se conforman a partir del trabajo de coautoría entre dos o más autores (Meadows, 1998). La comunicación científica supone el flujo de comunicación que se establece entre un grupo de investigadores -autores de investigaciones originales- con actividades y objetivos claros de trabajo (Borgman \& Furner, 2002). Para el análisis de dichas redes se utilizó el software VOSviewer. 1.6 (Van Eck \& Waltman, 2010; Kooij \& Waltman, 2019). Este software permite la elaboración de redes académicas para representar estructuras de conocimiento. El análisis de las redes de colaboración científica es un elemento crucial para el abordaje de las estructuras de conocimiento que emergen de las dinámicas de investigación de los estudios de familia-escuela. Las gráficas representadas constituyen las redes de coautoría que se establecen entre países y autores. Estas redes van a permitir agrupar, clasificar y determinar comunidades académicas al interior de los estudios de familia-escuela, así como establecer diferencias entre las dinámicas de producción científica indexada en las bases de datos. El conjunto de redes de colaboración constituye un vehículo de comunicación que permite dar cuenta de estructuras fundamentales de conocimiento (Esquivel, Carbonelli \& Irrazabal, 2011), donde tienen lugar las dinámicas de producción académica de los estudios en el área. Finalmente, se tomó una muestra de las investigaciones más citadas para llevar a cabo una revisión en detalle de la manera en la que se están definiendo los estudios sobre la relación familia-escuela.

\section{Resultados}

La producción académica de los estudios de la relación familia-escuela en español en la última década es de 564 artículos. La distribución temporal de esta producción no muestra una tendencia clara de crecimiento (figura 1); más bien, corresponde a una producción variable. Los años 2012 y 2017 corresponden a los periodos en los que se ha registrado la mayor alza de artículos, registrándose un umbral de 62 y 72 artículos, respectivamente. Por el contrario, los años 2008 y 2010 son los en los que se registra el menor número de publicaciones sobre esta temática, alcanzando solo 31 y 33 artículos, respectivamente.

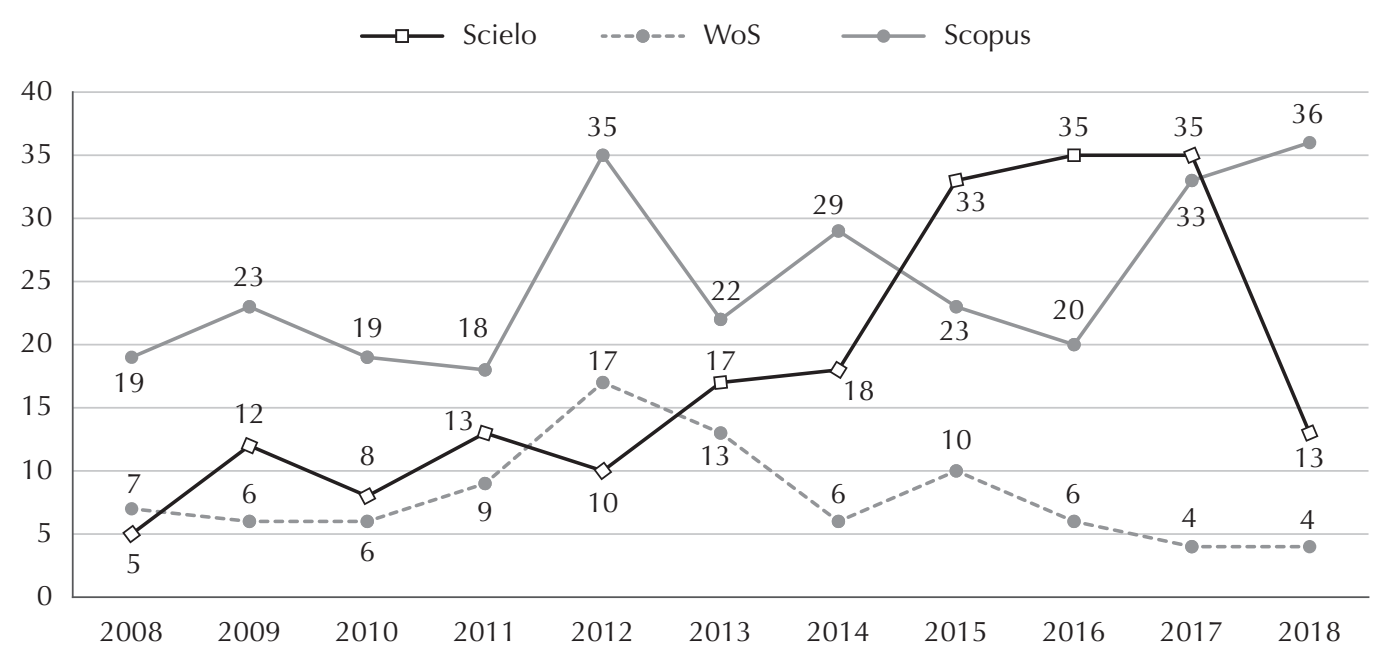

Figura 1. Producción académica de los estudios familia-escuela en la última década 2008-2018

Fuente: Elaboración propia. 
$\mathrm{Al}$ analizar esta década, el promedio de publicación de artículos por año no supera los 56 artículos. Sin embargo, al observar el promedio de publicación anual para cada una de las BBDD es posible constatar que hay diferencias en la producción académica de artículos en las tres BBDD consideradas. Mientras que en Scopus el promedio anual alcanza a 27,7 artículos en esta década, SciELO tiene una media de 19,9 y las bases indexadas de WoS solo alcanzan una media de 8,8 artículos anuales.

\section{Impacto de la producción académica}

En general, se constata un bajo impacto de la producción académica de esta temática. Una porción importante de esta producción $(42,9 \%, \mathrm{n}=242)$ nunca ha recibido una cita, mientras que 15,8\% ( $\mathrm{n}=89)$ de las investigaciones ha sido citada solo una vez. Así, 58,7\% de las publicaciones tuvo un impacto mínimo o nulo. Un cuarto de la restante producción sobre la temática tiene entre dos y cinco citas $(22,3 \%)$ y un 19\% de los artículos registra entre 6 y 36 citas para el período (tabla 1).

Tabla 1

Frecuencia de citación de acuerdo con las bases de datos. 2008-2018

\begin{tabular}{cccccccc}
\multirow{2}{*}{ Frecuencia } & \multicolumn{2}{c}{ SciELO } & \multicolumn{2}{c}{ Scopus } & \multicolumn{2}{c}{ Total } \\
\cline { 2 - 6 } & $\mathrm{N}$ & $\%$ & $\mathrm{~N}$ & $\%$ & $\mathrm{~N}$ & $\%$ & \\
\hline 0 & 123 & 61,8 & 92 & 33,2 & 27 & 30,7 & $42,9 \%$ \\
\hline 1 & 41 & 20,6 & 40 & 14,4 & 8 & 9,1 & $15,8 \%$ \\
\hline $2-5$ & 23 & 11,6 & 83 & 30,0 & 20 & 22,7 & $22,3 \%$ \\
\hline $6-10$ & 9 & 4,5 & 29 & 10,5 & 13 & 14,8 & $9,0 \%$ \\
\hline $11-15$ & 3 & 1,5 & 20 & 7,2 & 12 & 13,6 & $6,2 \%$ \\
\hline $16-20$ & -- & -- & 2 & 0,7 & 4 & 4,6 & $1,1 \%$ \\
\hline $21-30$ & -- & -- & 7 & 2,5 & 3 & 3,4 & $1,8 \%$ \\
\hline $31-36$ & -- & -- & 4 & 1,4 & 1 & 1,2 & $0,9 \%$ \\
\hline Total & 199 & 100 & 277 & 100 & 88 & 100 & 100 \\
\hline
\end{tabular}

Fuente: Elaboración propia.

Como se observa en la tabla 1, existen diferencias importantes en términos de citación al interior de las BBDD: Scopus es la BBDD con mayor impacto en los estudios de familia-escuela, seguida de SciELO y, finalmente, se encuentra WoS en su frecuencia de citación. No obstante, es importante hacer notar que, porcentualmente, los artículos publicados en Scopus y WoS tienen una tasa de citación (>2) muy superior a lo que pasa en SciELO. De manera inversa, SciELO duplica porcentualmente a Scopus y WoS, en los artículos que tienen solo una o ninguna citación. Cabe consignar que, en el periodo revisado, las políticas públicas de promoción científica en la mayoría de los países de origen tienden a promover la publicación de revistas indexadas en las dos primeras BBDD (WoS y Scopus) en desmedro de SciELO, lo que podría ser una de las variables que expliquen esta situación.

\section{¿Dónde se está llevando a cabo esta producción?}

Para responder a esta pregunta, los resultados se exponen en función de los países y comunidades académicas que vienen destacando en la producción académica de los estudios familia-escuela en función de las dinámicas de investigación en cada una de las BBDD. 


\section{Producción académica por países}

En total, son 27 los países que se registran. La figura 2 presenta las redes de coautoría por países que se identifican en la producción académica sobre estudios de familia-escuela, discriminado por las BBDD. Es importante mencionar que la red que se presenta en la figura 2 corresponde a los países de afiliación institucional de los investigadores que desarrollan los estudios de familia-escuela.

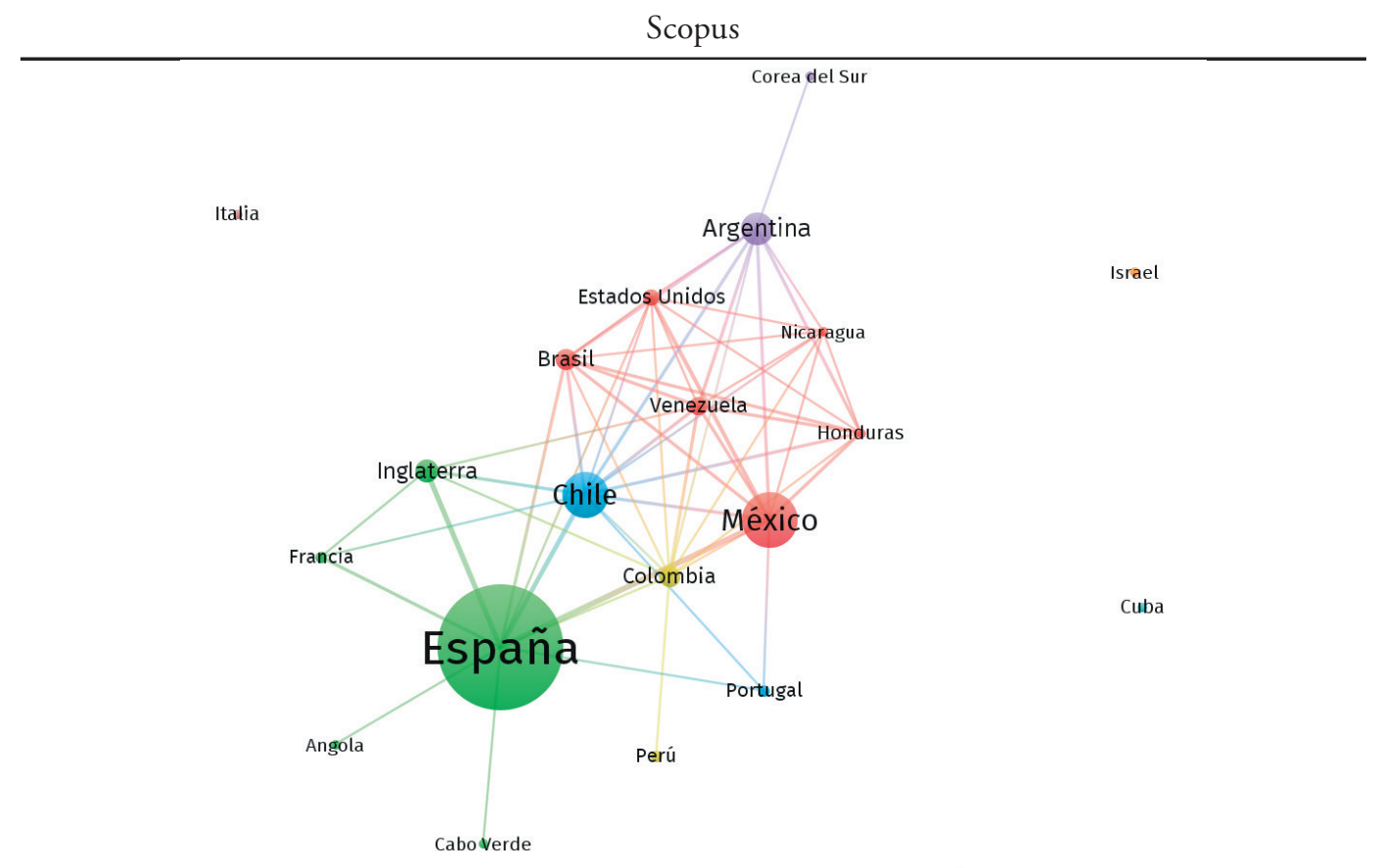

WoS

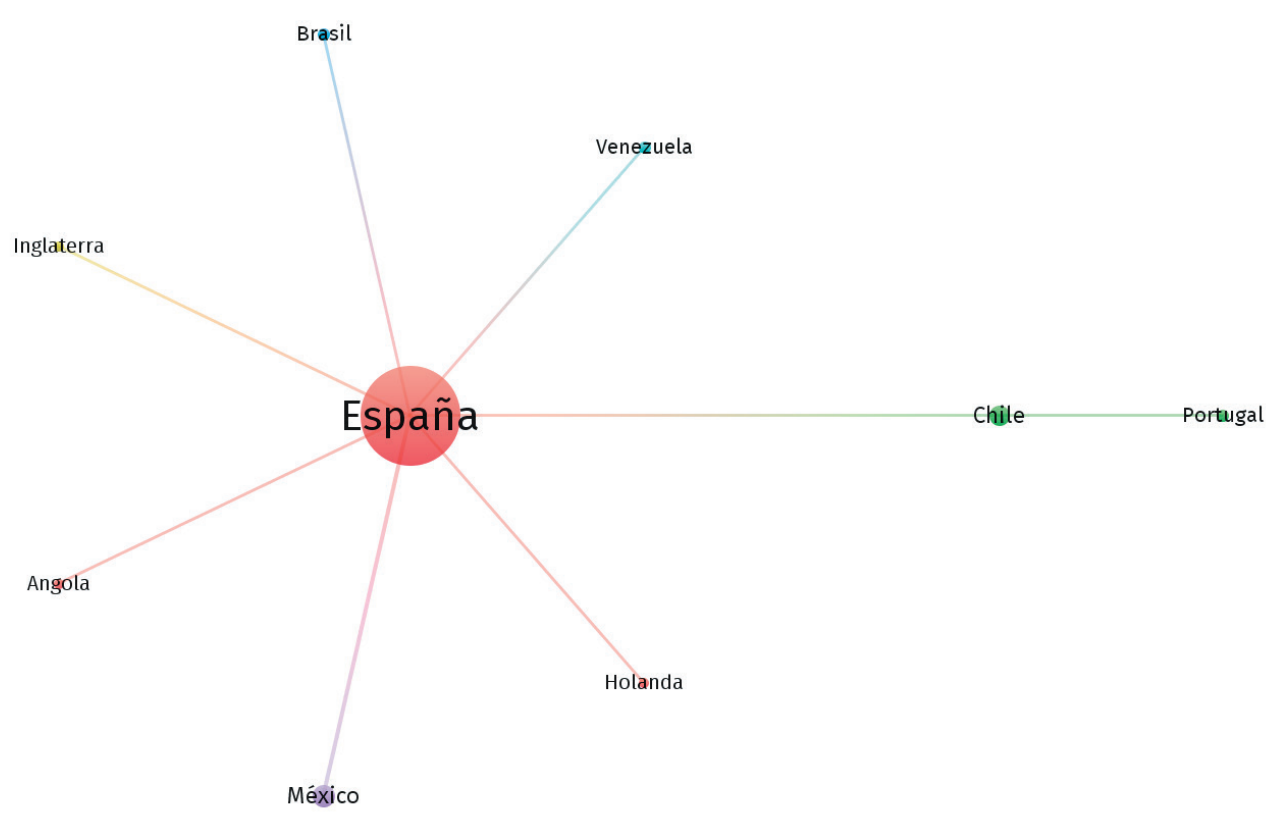


SciELO

\begin{tabular}{lll}
\hline Perú & Angola & \\
& Cuba & Uruguay
\end{tabular}

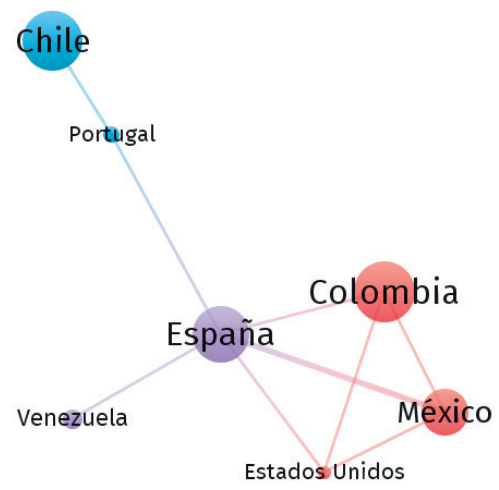

Figura 2. Redes de coautoría entre países. 2008-2018

Fuente: Elaboración propia.

La figura 2 expone las dinámicas de investigación en términos de coautoría por países en función de las BBDD, en ella destacan tres aspectos importantes, a saber: a) Scopus es la BBDD que mayor red de coautoría presenta, b) las investigaciones publicadas en Scopus parecen prever la conformación de comunidades académicas de investigadores conformadas en promedio por tres autores y c) las publicaciones en Scopus están siendo lideradas por investigadores españoles y se configuran relaciones de colaboración con investigadores especialmente de Latinoamérica, como México, Chile, Colombia y Argentina.

En segundo lugar, la producción académica que se registra en WoS no muestra la conformación de una red fuerte de colaboración. Las publicaciones se concentran casi exclusivamente en España y las relaciones con otros países de Europa y Latinoamérica es muy incipiente. En tercer lugar, SciELO muestra un patrón de colaboración entre los países de Latinoamérica importante y la contribución de investigadores españoles disminuye. Sin embargo, pese a que hay una participación visible de investigadores latinoamericanos, las redes están indicando la conformación de comunidades académicas de investigadores integradas en promedio por tres autores, donde no se evidencian redes de colaboración.

En términos generales, al revisar las dinámicas de investigación al interior de las tres BBDD, encontramos puntos de convergencia que es importante conocer. Por un lado, Latinoamérica representa el continente con mayor registro de países que contribuyen a la producción académica de los estudios de familia-escuela. La contribución latinoamericana en Scopus es de 57 \% ( $n=12)$, en WoS, de 44\% ( $n=4)$ y en SciELO, de 52\% ( $n=11)$.

Por otro lado, si bien es cierto que Latinoamérica constituye el continente con mayor representatividad de países, cuando revisamos las frecuencias de publicaciones en términos generales se encuentra que Europa es el continente que mayor aporta a la producción académica y España el país con más contribuciones. La producción académica europea representa 54,4\% ( $\mathrm{n}=320)$; la latinoamericana, 43,0\% $(\mathrm{n}=253)$; la norteamericana, 1,2\% $(\mathrm{n}=7)$; la africana, $0,9 \%(\mathrm{n}=5)$ y la asiática, $0,5 \%(\mathrm{n}=3)$. 
En este sentido, hay que llamar la atención al registro de investigaciones de habla hispana en regiones de África-Angola ( $\mathrm{n}=4)$, Cabo Verde $(\mathrm{n}=1)$ - y Asia: Israel, Taiwán y Corea del Sur con una contribución cada uno. Los cinco países con mayores contribuciones son: España ( $n=292)$; México ( $n=66)$; Chile ( $n=66)$; Argentina $(\mathrm{n}=39)$, y Colombia $(\mathrm{n}=37)$. La contribución de estos países representa 84,5\% (497) de la producción académica total. Sin embargo, es importante destacar que son estos mismos países los que lideran la producción académica general de la ciencia en idioma español (Gómez-García, Ramiro, Ariza \& Granados, 2012).

\section{Comunidades académicas}

El registro de autores vinculados con la producción académica de los estudios familia-escuela es de 1479. El número varía de acuerdo con el registro de las bases de datos del siguiente modo: Scopus 48,5\% (n=708); WoS $17 \%(\mathrm{n}=248)$ y SciELO 34,5\% ( $\mathrm{n}=503)$. La figura 3 expone la red de coautoría que se teje entre los autores al interior de los estudios de familia-escuela que, al menos, han contribuido con dos investigaciones.

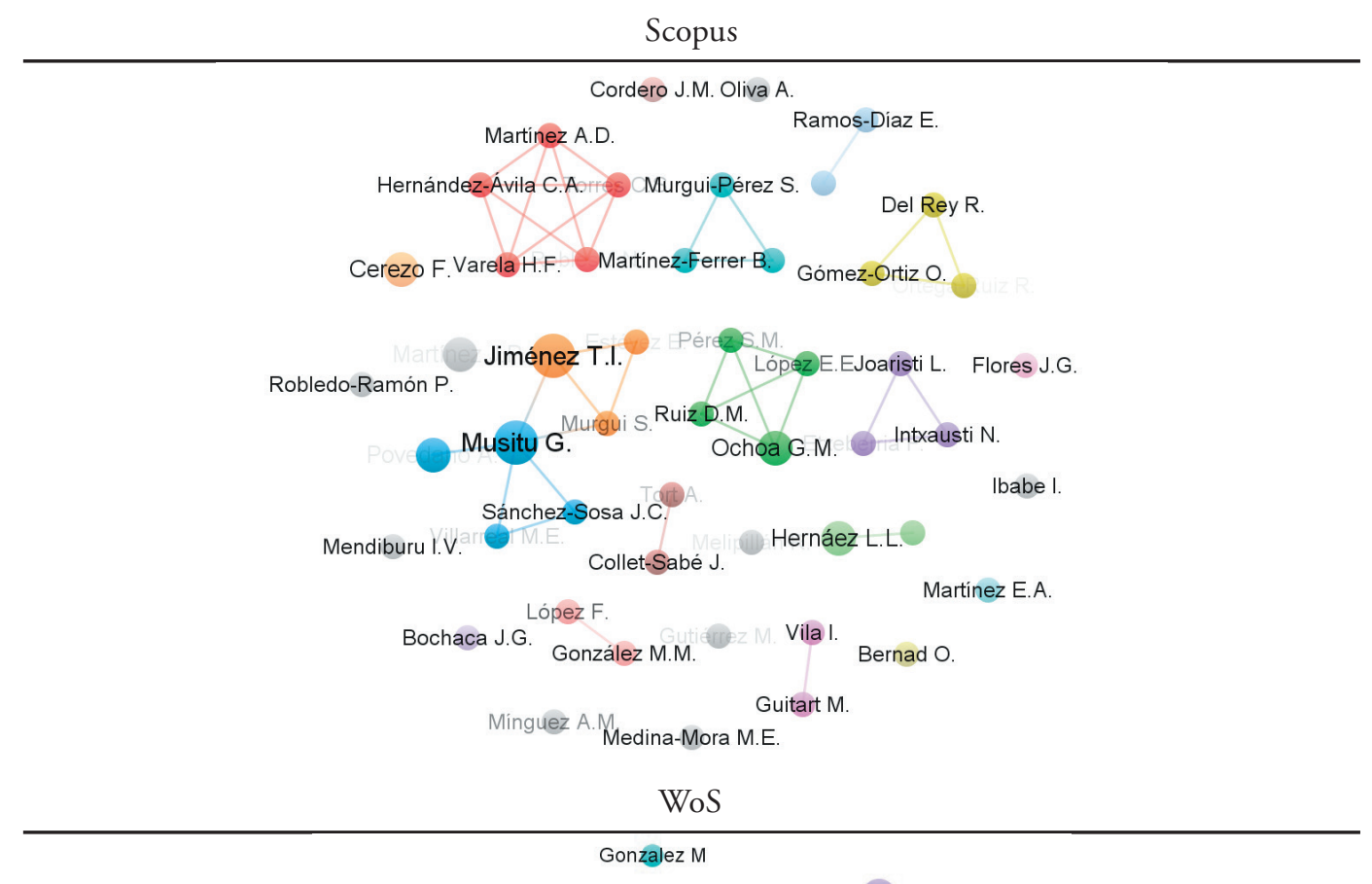

Gil-Flores $J$

Ibabe I 


\section{SciELO}

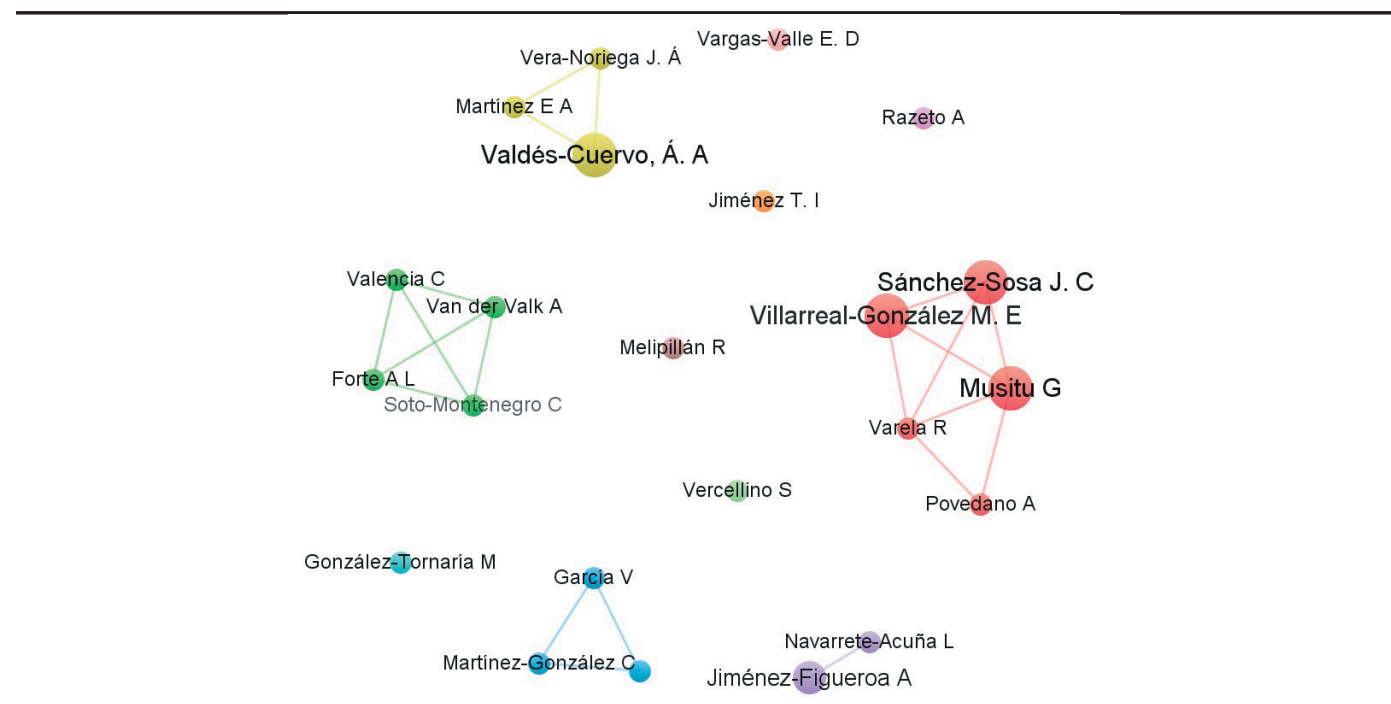

Figura 3. Redes de coautoría entre investigadores en los estudios Familia-Escuela. 2008-2018

Fuente: Elaboración propia.

La figura 3 representa el detalle de las comunidades académicas de investigadores que vienen trabajando colaborativamente. Por lo tanto, como se evidenció en la red de colaboración por países, Scopus es la BBDD donde se configuran mayores redes de colaboración. Si bien se aprecia la conformación de nichos de investigación conformados en promedio por tres autores, es importante mencionar que las comunidades que la componen no muestran un vínculo de trabajo colaborativo con otras comunidades. La tabla 2 expone en detalle el país de origen de los investigadores que conforman comunidades académicas al interior de los estudios de familia-escuela.

Tabla 2

Afliación institucional y paises de procedencia de los investigadores en estudios de familia-escuela

\begin{tabular}{cccc} 
Base de datos & Investigador & Institución & País \\
\hline & Hernández-Ávila, C. A. & Universidad Nacional Autónoma de México & México \\
\cline { 2 - 4 } & Martínez, A. D. & Universidad Nacional Autónoma de México & México \\
\hline Robles, J. N. & Universidad Nacional Autónoma de México & México \\
\cline { 2 - 4 } Scopus & Torres, C. S. & Universidad Nacional Autónoma de México & México \\
\cline { 2 - 4 } & Varela, H. F. & Universidad Nacional Autónoma de México & México \\
\cline { 2 - 4 } & López, E. E. & Universidad de Valencia & España \\
\cline { 2 - 4 } & Ochoa, G. M. & Universidad Pablo Olavide & España \\
\hline Pérez, S. M. & Universidad Católica San Vicente Mártir & España \\
\hline Ruiz, D. M. & Universidad de Valencia & España \\
\hline Musitu, G. & Universidad de Pablo Olavide & España \\
\hline
\end{tabular}




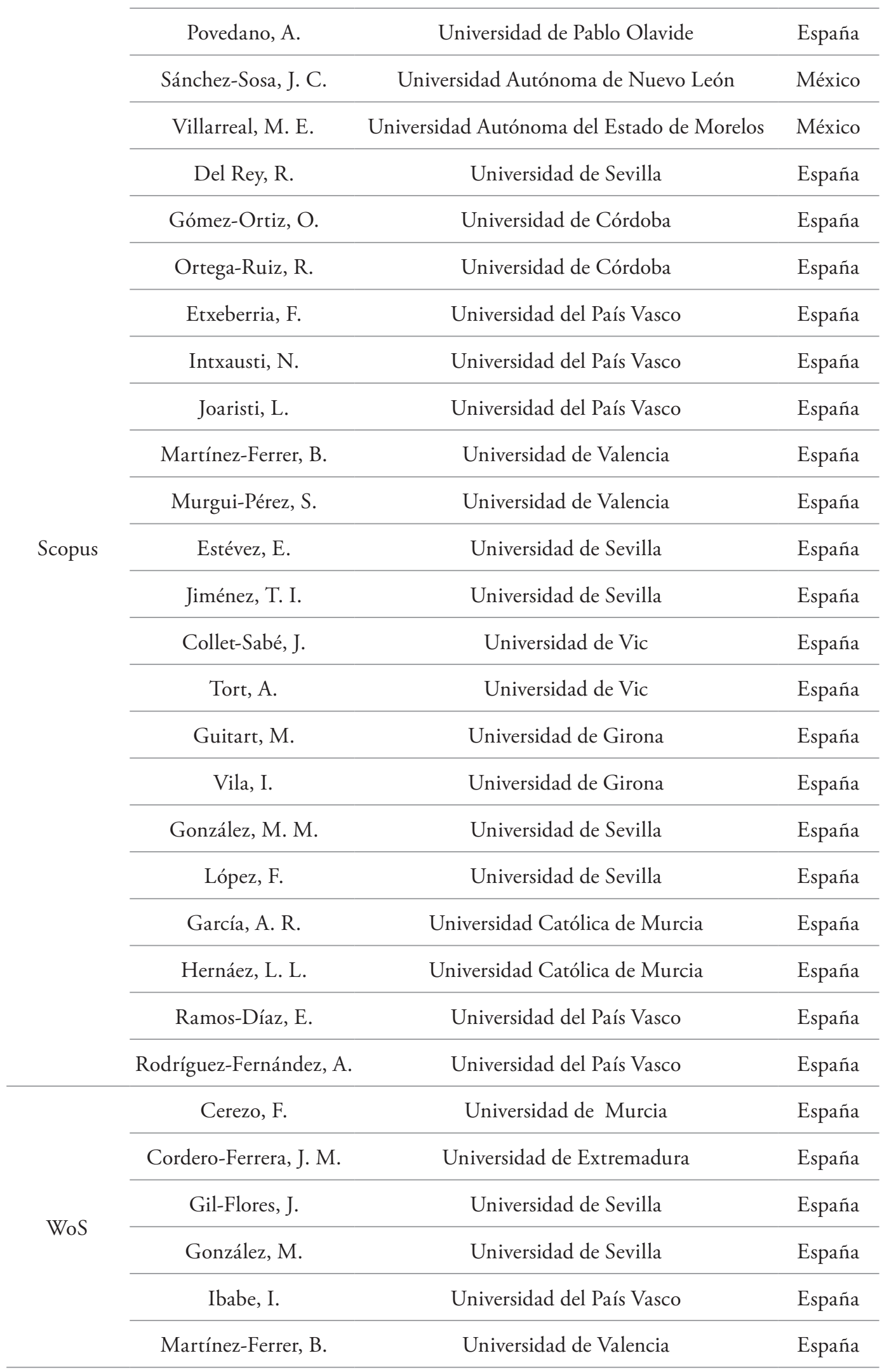




\begin{tabular}{|c|c|c|c|}
\hline \multirow{19}{*}{ Scielo } & Varela, R. & Universidad Pablo de Olavide & España \\
\hline & Forte, A. L. & Universidad Católica Boliviana Tarija & Bolivia \\
\hline & Soto-Montenegro, C. & Universidad Católica Boliviana Tarija & Bolivia \\
\hline & Valencia, C. & Universidad Católica Boliviana Tarija & Bolivia \\
\hline & Van der Valk, A. & Universidad Católica Boliviana Tarija & Bolivia \\
\hline & García, V. & Universidad Nacional Autónoma de México & México \\
\hline & Martínez-González, C. & Facultad Latinoamericana de Ciencias Sociales & México \\
\hline & Ramírez-Pérez, J. A. & Universidad Autónoma del Estado de Morelos & México \\
\hline & Martínez, E. A. & Instituto Tecnológico Superior de Cajeme & México \\
\hline & Valdés-Cuervo, Á. A & Instituto Tecnológico de Sonora & México \\
\hline & Vera-Noriega, J. Á. & $\begin{array}{c}\text { Centro de Investigación en Alimentación y } \\
\text { Desarrollo A.C. }\end{array}$ & México \\
\hline & Jiménez-Figueroa, A. & Universidad de Talca & Chile \\
\hline & Navarrete-Acuña, L. & Instituto Diego Portales & Chile \\
\hline & González-Tornaría, M. & Universidad Católica del Uruguay & Uruguay \\
\hline & Jiménez, T. I. & Universidad de Zaragoza & España \\
\hline & Melipillán, R. & Universidad de Concepción & Chile \\
\hline & Razeto, A. & Pontificia Universidad Católica de Chile & Chile \\
\hline & Vargas-Valle, E. D. & El Colegio de la Frontera Norte & México \\
\hline & Vercellino, S. & Universidad Nacional de Río Negro & Argentina \\
\hline
\end{tabular}

Fuente: Elaboración propia.

En Scopus se observa la conformación de ocho grupos de investigación que marcan una línea de producción en los estudios de familia-escuela. El clúster 1 (color rojo) liderado por C.A. Hernández-Ávila et al.; los clústeres 2 y 3 (color azul y naranja) liderados por G. Musito G. et al. y E. T. Jiménez et al. constituyen las comunidades más representativas.

Por otro lado, como se puede apreciar en la figura 3, no se presentan vínculos fuertes de colaboración entre investigadores en la BBDD de WoS; el carácter de las publicaciones en esta BBDD está dado por contribuciones de un solo autor. Por último -a diferencia de WoS-, SciELO tiene una importante dinámica de investigación en red. Se decantan tres comunidades académicas que se vienen consolidando en los estudios de familia-escuela, a saber: el clúster 1 (color rojo), representando por G. Musito, M. E. Villarreal-González, J. C. Sánchez-Sosa; el clúster 2 (color amarillo) representado por Á. A. Valdés-Cuervo, J. A. Vera-Noriega y E. A. Martínez; el clúster 3 (color verde) representado por A. Van der Valk, C. Valencia, A. L. Forte y C. Soto-Montenegro.

Finalmente, cuando se revisa la frecuencia de autores que firman las investigaciones sobre los estudios de familia-escuela, se observa que $72,5 \%(\mathrm{n}=408)$ de la producción académica se registra con un intervalo de uno a tres autores por artículo de la siguiente manera: artículos firmados por un autor, 19,5\% (n=110); artículos firmados por dos autores, 27,2\% ( $\mathrm{n}=153)$ y artículos firmados por tres autores, $25,8 \%(\mathrm{n}=145)$. La tabla 3 presenta la frecuencia de autores firmantes en las investigaciones sobre los estudios familia-escuela. 
Tabla 3

Frecuencia de autores firmantes en los estudios de familia-escuela. 2008-2018

\begin{tabular}{cccc} 
Frecuencia autores & No. Artículos & Total citas & Promedio citas \\
\hline 1 & 110 & 70 & 0,6 \\
\hline 2 & 153 & 79 & 0,5 \\
\hline 3 & 145 & 89 & 0,6 \\
\hline $4-6$ & 148 & 103 & 0,7 \\
\hline $7-10$ & 7 & 4 & 0,6 \\
\hline 29 & 1 & -- & 0 \\
\hline Total & 564 & 345 & 3,0 \\
\hline
\end{tabular}

Fuente: Elaboración propia.

Como se puede apreciar en la tabla 3, 72,5\% (n=408) de la producción académica está firmada por un rango de uno a tres autores como lo muestra el párrafo anterior y $27,5 \%(\mathrm{n}=156)$ corresponde a la producción académica restante está distribuida en un rango mayor de coautorías. Sin embargo, este aspecto no permite reflejar una tendencia clara que permita indicar que los estudios de familia-escuela se enmarquen en una lógica de la híper autoría, características de las nuevas dinámicas de producción científica (Cronin, 2001). En ese sentido, es importante mencionar que la cantidad de autores no está relacionada con un aumento de las citas, a diferencia de otros estudios que presentan que el incremento de la citación obedece a la colaboración entre autores (Garner, Hirsch, Albuquerque \& Fargen, 2018).

\section{Autores representativos}

El volumen de artículos por investigador en los estudios sobre familia-escuela no supera los cuatro artículos. Esta situación es similar en cada una de las BBDD consultadas. Esto permite mostrar que la autoría al interior de los estudios de familia-escuela es un aspecto muy naciente de cara a su consolidación. La tabla 4 permite conocer a los autores más representativos en los estudios de familia-escuela en función del volumen de artículos.

Tabla 4

Autores representativos de estudios sobre familia-escuela en español. 2008-2018

\begin{tabular}{ccc} 
Autor & No. art. & Base de datos \\
\hline Jiménez, Teresa & 4 & Scopus \\
\hline Sánchez-Sosa, Juan Carlos & 4 & SciELO \\
\hline Villarreal-González, María Elena & 4 & SciELO \\
\hline Cerezo, Fuensanta & 3 & WoS \\
\hline Gil-Flores, Javier & 3 & WoS \\
\hline Martínez-Ferrer, Belén & 3 & WoS \\
\hline Musitu, Gonzalo & 3 & Scopus \\
\hline Hernáez, Lara L. & 3 & Scopus \\
\hline Parra Martinez, Joaquín & 3 & Scopus \\
\hline Povedano, Amapola & 3 & Scopus \\
\hline Jiménez-Figueroa, Andrés & 3 & SciELO \\
\hline Valdés-Cuervo, Ángel & 3 & SciELO
\end{tabular}

Nota: Los artículos en más de una base de datos fueron eliminados de la base de menor indexación (ver apartado metodológico).

Fuente: Elaboración propia. 
Entre aquellos autores distintivos expuestos en la tabla 4, 58\% de ellos son hombres y 42\%, mujeres. Estos autores representan 7,4\% $(n=42)$ de la producción académica en el área. Este aspecto es un ejemplo que permite explicar la heterogeneidad y dispersión de las comunidades académicas que se vienen conformando al interior de este campo en el contexto hispanoamericano.

\section{Revistas donde se está publicando}

Un número importante de revistas revela el flujo de comunicación científica ante la producción académica de los estudios de familia-escuela. Un total de 564 artículos publicados en 184 revistas especializadas en diversas áreas de las ciencias sociales, educación y psicología ratifica la importancia de las revistas como canal de comunicación científica para las comunidades académicas (Buela-Casal \& López-López, 2005; Madrid, Jiménez-Fanjul, LeónMantero \& Maz-Machado, 2017; Salas et al., 2019). Además, consolidan su responsabilidad de optimizar la visibilidad en la producción académica local, regional y nacional (Cudina, Millán \& Ossa, 2017; Polanco, Beria \& Klappenbach, 2017; Polanco-Carrasco, Gallegos, Salas \& López-López, 2017).

El número de revistas varía de acuerdo con su indexación en las bases de datos de la siguiente manera. En Scopus se encuentran registradas $(n=103)$ revistas, en WoS, $(n=20)$ y en SciELO, $(n=89)$. La figura 4 muestra las diez revistas que más publican en los estudios de familia-escuela. Es necesario mencionar que estas revistas proporcionan $36,5 \%(\mathrm{n}=206)$ de la producción académica.

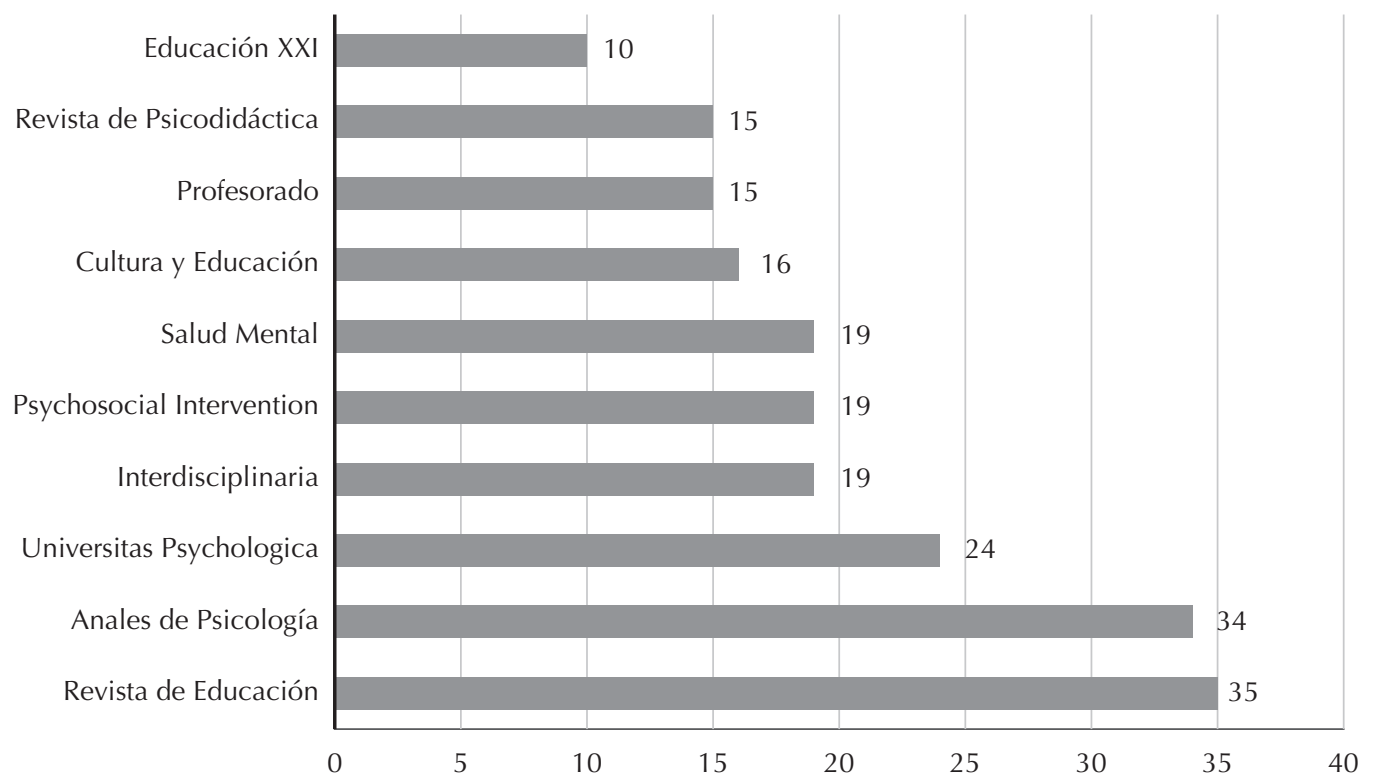

Figura 4. Revistas representativas en los estudios de familia-escuela. 2008-2018

Fuente: Elaboración propia.

La figura 4 muestra que las publicaciones en estudios de familia-escuela se ubican en revistas de distintas áreas de especialización, distribuidas en un 50\% $(\mathrm{n}=5)$ en revistas del área psicosocial y de salud mental, donde es frecuente el uso de escalas de medición del clima social familiar, escolar y de participación. Esto es seguido de un $50 \%(n=5)$ en revistas de cultura y educación, centradas en estudios educativos con perspectivas culturales, según el contexto. Es importante destacar que no existe evidencia de que la mayor frecuencia en publicaciones observadas en el gráfico anterior sea a causa de ediciones especiales sobre la materia (Special Issue). 


\section{¿Qué están abordando los trabajos más citados?}

En la tabla 5 se pueden identificar los 10 artículos más citados, adscritos a Scopus y WoS, a los cuales se adicionan los dos artículos más citados en SciELO, para un total de 12 artículos seleccionados que permiten identificar las principales variables contenidas en los estudios.

Tabla 5

Investigaciones más citadas que abordan la relación familia-escuela 2008-2018

Autor/Año/País

\begin{tabular}{|c|c|c|c|}
\hline Autor/Año/País & Título del artículo & Citas & Base de datos \\
\hline $\begin{array}{l}\text { Cordero, Crespo y Pedraja. } \\
\text { (2013) - España }\end{array}$ & $\begin{array}{l}\text { Logros educativos y determinantes en } \\
\text { PISA: una encuesta de literatura española }\end{array}$ & 36 & Scopus \\
\hline $\begin{array}{l}\text { Jiménez, Musitu y Murgui. } \\
\text { (2008)- España }\end{array}$ & $\begin{array}{l}\text { Funcionamiento familiar y uso de } \\
\text { sustancias en adolescentes. El papel } \\
\text { mediador de la autoestima }\end{array}$ & 33 & Scopus \\
\hline $\begin{array}{l}\text { Castaño, Gómez y Bouachra. } \\
\text { (2008) - España }\end{array}$ & $\begin{array}{c}\text { Población inmigrante y escuela en España: } \\
\text { un balance de investigación }\end{array}$ & 31 & Scopus \\
\hline $\begin{array}{l}\text { Ruiz, López, Pérez y Ochoa. } \\
\text { (2009) - España }\end{array}$ & $\begin{array}{l}\text { Relación entre el entorno familiar y el } \\
\text { escolar: el papel de la empatía, la actitud } \\
\text { ante la autoridad y la violencia. }\end{array}$ & 29 & Scopus \\
\hline $\begin{array}{l}\text { Povedano, Hendry, Ramos y Varela. } \\
\text { (2011) - España }\end{array}$ & $\begin{array}{l}\text { Victimización escolar: ambiente familiar, } \\
\text { autoestima y satisfacción con la vida desde } \\
\text { una perspectiva de género }\end{array}$ & 26 & Scopus \\
\hline $\begin{array}{l}\text { Cerezo, Sánchez, Ruiz y Arense. } \\
\text { (2015) - España }\end{array}$ & $\begin{array}{c}\text { El papel de los adolescentes y } \\
\text { preadolescentes en el acoso escolar y su } \\
\text { relación con el clima social y los estilos de } \\
\text { crianza }\end{array}$ & 23 & WoS \\
\hline $\begin{array}{l}\text { Martínez-Ferrer, Murgui-Pérez, } \\
\text { Musitu-Ochoa y Monreal-Gimeno. } \\
\text { (2008) - España }\end{array}$ & $\begin{array}{l}\text { El rol del apoyo parental, las actitudes } \\
\text { hacia la escuela y la autoestima en la } \\
\text { violencia escolar en adolescentes }\end{array}$ & 22 & Scopus \\
\hline $\begin{array}{c}\text { Gázquez, Pérez y Carrión. (2011) } \\
\text { - España, Hungría, Austria y } \\
\text { República Checa }\end{array}$ & $\begin{array}{l}\text { Clima escolar y resolución de conflictos } \\
\text { según los estudiantes: un estudio europeo }\end{array}$ & 22 & WoS \\
\hline $\begin{array}{l}\text { Gómez-Ortiz, Del Rey, Casas y } \\
\text { Ortega-Ruiz. (2014) - España }\end{array}$ & Estilos parentales e implicación en bullying & 21 & Scopus \\
\hline $\begin{array}{l}\text { Hernando, Oliva y Pertegal. } \\
\text { (2012) - España }\end{array}$ & $\begin{array}{l}\text { Variables familiares y rendimiento } \\
\text { académico en la adolescencia }\end{array}$ & 21 & Scopus \\
\hline $\begin{array}{l}\text { Sánchez-Sosa, Villarreal-González, } \\
\text { Musitu y Martínez. (2010) - España }\end{array}$ & $\begin{array}{l}\text { Ideación suicida en adolescentes: un } \\
\text { análisis psicosocial }\end{array}$ & 13 & SciELO \\
\hline $\begin{array}{l}\text { Uribe, Orcasita y Aguillón. } \\
\qquad(2012) \text { - Colombia }\end{array}$ & $\begin{array}{c}\text { Bullying, redes de apoyo social y } \\
\text { funcionamiento familiar en adolescentes } \\
\text { de una institución educativa de Santander, } \\
\text { Colombia }\end{array}$ & 10 & SciELO \\
\hline
\end{tabular}

Fuente: Elaboración propia. 
De los estudios relacionados en la tabla anterior, 92\% son desarrollados en España (en diferentes ciudades del país) y $8 \%$, en Colombia. Al indagar en el contenido de estos estudios respecto de la referencia conceptual de la relación entre familia-escuela, solo uno de ellos hace alusión a que la relación familia-escuela es considerarla como uno de los factores más importantes en la integración escolar de este alumnado (García-Castaño, Rubio-Gómez \& Bouachra, 2008), referencia que si bien no define el concepto, contextualiza la relación directamente como importante. Los demás estudios lo hacen de forma indirecta sin acercarse a definiciones, cuando por ejemplo expresan que las investigaciones se centran en el estudio de la relación conjunta de los contextos familiar y escolar, sin definirlo.

Las investigaciones presentan diversos objetos de estudio relacionados con familia-escuela y analizan situaciones asociadas al funcionamiento familiar, el clima familiar, el contexto familiar frente a las situaciones que se viven en espacios educativos como rendimiento académico, autoestima, consumo de sustancias psicoactivas, ideación suicida, acoso escolar, incorporación de escolares en condiciones migrantes, clima escolar. Estos estudios entienden a la familia como una variable explicativa de bienestar o malestar del estudiante y su relación con la escuela.

En estas investigaciones, los objetos de estudio han sido analizados mediante variables, entre las que se encuentran la medición del clima sociofamiliar y escolar, como un aspecto fundamental cuando se quiere posicionar y cuantificar las características de dicha relación. Si bien los estudios no lo presentan directamente, sí es posible considerar su utilidad para ubicar y posicionar la relación entre familia-escuela, valorando las características incluidas en dichas escalas. Asimismo, existe la inclusión que hacen algunos estudios de la valoración de los estilos de crianza, centrados en estilos educativos parentales, que conciben la participación de la familia como una característica importante desde su vinculación en los procesos escolares con adolescentes. Estas escalas son formuladas desde el área disciplinar de la psicología.

\section{Apuntes generales de las investigaciones sobre familia- escuela en el contexto latinoamericano}

Al revisar en detalle el panorama de las comunidades académicas que se conforman a través de las coautorías entre investigadores, podemos observar que $43 \%$ se corresponde a investigadores latinoamericanos. Como se puede apreciar, los nichos de trabajo se conforman en promedio por tres investigadores, sin evidencia clara de vínculos de trabajo colaborativo con otras comunidades de investigación. La afiliación institucional y los países de procedencia de quienes integran los nichos de investigación en los países hispanohablantes del continente americano quedan representados así: en Scopus, México, con cinco autores; en WoS no se encuentra autoría; en Scielo, 11 autores (Bolivia, 4; Chile, 4; Uruguay 1; Argentina 1). En el caso de Chile, se evidencian las contribuciones de académicos como Andrés Jiménez-Figueroa, Lucina Navarrete-Acuña, Roberto Melipillán y Alicia Razeto.

Es importante mencionar que, dados los criterios de inclusión seleccionados para los heurísticos, es posible que algunos autores con una trayectoria importante que han contribuido en el avance de las investigaciones sobre familia-escuela en el contexto chileno, como Verónica Gubbins, Gabriel Otero, Héctor Cárcamo, Alejandra Santana y Taly Reininger, no se reconozcan en estos mapas de redes, ya que las palabras clave en el campo no están estandarizadas, lo que supone una dispersión de los términos utilizados.

\section{Conclusiones}

Este trabajo permitió identificar la producción científica en el área de estudios que abordan la relación familiaescuela. De este estudio se desprenden algunos hallazgos importantes. 
El desarrollo de la producción académica en los estudios de la relación familia-escuela en el periodo analizado (2008-2018) no presenta una muestra clara de crecimiento, ya que su mayor alza ocurre en 2012, con 62 publicaciones, y en 2017, con 67. Esto muestra una producción fluctuante dada por incrementos y disminuciones en la publicación, sin una trayectoria estable. Esto invita a consolidar el campo temático mediante las investigaciones en idioma español.

En cuanto a la procedencia de las publicaciones y de los autores, se identificó que el mayor lugar de producción se encuentra en Europa, con una representatividad de 54,5\% de los estudios y, en segundo lugar, en Latinoamérica, con 43\%. Los principales países, en orden de producción, son España, México, Chile, Argentina y Colombia. No obstante, se identifican otros como Francia, Portugal, Reino Unido y Estados Unidos, con publicaciones en español, lo cual podría tener relación con la presencia de autores hispanohablantes en dichos territorios (Ávila-Toscano, 2017). De estos, 58\% de los autores son hombres y 42\%, mujeres, lo que es superior al 30\% del promedio mundial de mujeres en ciencias (Unesco, 2020).

Por otra parte, la conformación de las redes de investigación en este campo permitió identificar comunidades académicas, donde $72,5 \%$ de las publicaciones cuentan con un intervalo de uno a tres autores y $27,5 \%$, con cuatro autores o más. Los autores más representativos no superan los cuatro artículos publicados, lo que probablemente explique que la autoría es un aspecto naciente en este campo. Esto posiblemente permita justificar la dispersión que presentan las comunidades académicas, donde también se identifican autores que publican de forma independiente y no forman redes de colaboración científica en el campo estudiado. En la autoría de más de dos autores, se identifica una tendencia a citarse entre ellos, lo cual puede explicar las razones de que existan las comunidades aisladas. Lo anterior constituye en un desafío de mayor colaboración para los equipos de investigación en el área.

Con respecto a las diferencias existentes en la citación al interior de las BBDD, encontramos que Scopus es la BBDD con mayor impacto en los estudios de familia-escuela, seguida de SciELO; por último, se encuentra WoS en su frecuencia de citación. Estos resultados son coincidentes con Bravo (2013), quien muestra que Scopus es la BBDD de literatura científica con mayor cobertura en todas las ramas de la ciencia y concluye que Scopus es una BBDD científica y herramienta con mayor cobertura que WoS.

En la revisión de los trabajos más citados, el análisis muestra que dichos estudios no hacen definición del constructo que se indaga, relación familia-escuela. Los investigadores reconocen la importancia de los contextos familiares y escolares y su relación, mas no lo definen. En este sentido puede considerarse que, en las investigaciones, la relación familia-escuela como concepto está implícitamente descrito en el desarrollo del estudio y no consideran necesario plantear una definición explícita del concepto. Por ello, para las futuras investigaciones se propone declarar un posicionamiento del concepto, que permita comprender las miradas teóricas y epistemológicas con las cuales se abordan los asuntos de dicha relación.

Algunas limitantes de este estudio, a la hora de identificar equipos y autores relevantes de la investigación latinoamericana y europea en el campo de estudios de escuelas y familias, dicen relación con que: a) al dar cuenta exclusivamente de la publicación en español no se incluye la investigación que equipos hispanohablantes puedan haber publicado en otros idiomas, especialmente; b) al excluir las publicaciones en portugués se invisibiliza el trabajo realizado en Brasil, tales como el producido por el núcleo de estudios de familia, escuela y sociedad (NESFE-UFOP) o el Observatório Sociológico Família Escola (OSFE-UFMG), quienes llevan ya un tiempo investigando en el campo; c) no se consideran otros productos de investigación como proceedings, tesis, libros o artículos publicados en otras bases de datos; y d) las palabras clave buscaron ser lo suficientemente amplias ante la diversidad de investigaciones en el área. Sin embargo, estamos en presencia de una dispersión en el uso de palabras clave, muchas veces utilizadas por una única vez. Este punto no solo es una dificultad para la realización de la revisión, sino que dificulta tanto la visibilización de las investigaciones como su integración en un estudio bibliométrico como este, con criterios de búsqueda tan específicos. 
En términos de la fortaleza del estudio, este permite un primer acercamiento a la comprensión del campo de estudios de la relación familia-escuela y permite visualizar comunidades científicas en los países hispanohablantes en promedio de tres autores, donde no se evidencian redes de colaboración con otras comunidades. Esto coincide con otras tendencias, como en Ciencias Sociales, donde se observa una ausencia de híper autoría como característica en las lógicas actuales de producción y circulación de conocimiento (Cronin, 2001).

Adicionalmente, nos permite identificar que -desde el interés de comprensión del campo de estudios de la relación familia-escuela- se abren nuevos caminos de investigación. En primer lugar, definir y posicionar el constructo referido a la relación familia-escuela. En segundo lugar, afianzar la construcción de comunidades de investigación y producción en el campo de estudios de la relación familia-escuela que se traduzca en publicación, pudiendo ser un desafío para pensar la relación familia-escuela desde Latinoamérica. En tercer lugar, será importante realizar un estudio que incorpore índices de colaboración como Subramanyam (1983), Lawani (1981) y otras leyes de concentración de la producción, como Lotka (1926), todo lo cual permitirá comprender con mayor detalle cuestiones asociadas a la colaboración y la productividad en el campo familia-escuela.

Por último, resulta indiscutible señalar la importancia que tienen los estudios bibliométricos para conocer el estado de la cuestión de un tema y su comprensión global (Gálvez, 2016; Kock, Tulla \& Azevedo, 2016). La información que aporta este trabajo ofrece un marco representativo de cómo se ha investigado el campo de la relación familia-escuela desde 2008 a 2018, como base para futuras investigaciones, así como aquellos indicadores que presenta el estudio, la posibilidad de abordar este campo de estudios desde otras líneas teóricas, incluir la revisión en otros idiomas, así como la imperiosa necesidad de construir comunidades académicas que fortalezcan el campo de investigación, para consolidar y fortalecer las investigaciones.

Financiamiento: Esta investigación ha sido financiada por la Asociación Nacional de Investigación y Desarrollo (ANID) a través del Proyecto Fondecyt Regular 1181925 y Beca Doctorado Nacional 2021- ANID Folio N²1212239.

El artículo original fue recibido el 24 de septiembre de 2020

El artículo revisado fue recibido el 3 de mayo de 2021

El artículo fue aceptado el 22 de junio de 2021

\section{Referencias}

Abramo, G. \& D’angelo, C. A. (2011). Evaluating research: from informed peer review to bibliometrics. Scientometrics, 87(3), 499-514. https://doi.org/10.1007/s11192-011-0352-7

Ávila-Toscano, J. H. (2017). El exilio como campo de estudios en ciencias sociales: análisis bibliométrico. Revista Colombiana de Ciencias Sociales, 8(1), 172-188. https://doi.org/10.21501/22161201.1947

Ávila, M. \& Moreno, L. (2020). La Relación Familia - Escuela: Producción Académica del Quinquenio 2012-2017 (Tesis doctoral, Universidad de la Sabana). Recuperada de http://hdl.handle.net/10818/39638

Baquedano-López, P., Alexander, R. A. \& Hernández, S. J. (2013). Equity Issues in Parental and Community Involvement in Schools: What Teacher Educators Need to Know. Review of Research in Education, 37(1), 149-182. https://doi.org/10.3102/0091732X12459718

Bravo, V. A. (2013). Comparativo de dos bases de datos de literatura científica. Revista Fitotecnia Mexicana, 36(2), 93-94. Recuperado de https://www.revistafitotecniamexicana.org/documentos/36-2/EDITORIAL.pdf

Bolívar, A. (2006). Familia y escuela: dos mundos llamados a trabajar en común. Revista de educación, 339(1), 119-146. Recuperado de http://hdl.handle.net/11162/68682 
Borgman, C. L. \& Furner, J. (2002). Scholarly communication and bibliometrics. Annual Review of Information Science and Technology, 361(1), 2-72. https://doi.org/10.1002/aris.1440360102

Buela-Casal, G. \& López-López, W. (2005). Evaluación de las revistas científicas iberoamericanas de psicología. iniciativas y estado actual. Revista Latinoamericana de Psicología, 37(1), 211-217.

Cárcamo, H. \& Garreta, J (2020). Representaciones sociales de la relación familia-escuela desde la formación inicial del profesorado. Revista REDIE, 22, https://doi.org/10.24320/redie.2020.22.e11.2406

Carmona-Santiago, J., García, M., Máiquez, M. L. \& Rodrigo, M. J. (2019). El impacto de las relaciones entre la familia y la escuela en la inclusión educativa de alumnos de etnia gitana. Una revisión sistemática. Multidisciplinary Journal of Educational Research 9(3), 319-348. https://doi.org/10.17583/remie.2019.4666

Castelli, S. \& Pepe, A. (2008). School-parents relationships: A bibliometric study on 40 years of scientific publications. International Journal about Parents in Education, 2(1), 1-12. Recuperado de http://web.archive.org/web/20200711170822/http://www.ernape.net/ejournal/index.php/IJPE/article/view/67

Castrillón-Correa, E. M., Cossio-Bolaños, M., Cudina, J. N., Gómez-Campos, R. \& Precht, A. (2020). Agencia de estudiantes y familias en la adaptación sociocultural a nuevos contextos escolares: Una revisión sistemática. Espacios, 41(20), 169-184. Recuperado de https://www.revistaespacios.com/a20v41n20/20412014.html

Chávez, P. \& Vergara, A. (2017). Ser niño y niña en el Chile de hoy. La perspectiva de sus protagonistas acerca de la infancia, la adultez y las relaciones entre padres e hijos. Santiago, Chile: Ceibo.

Cronin, B. (2001). Hyperauthorship. A Postmodern Perversion or Evidence of a Structural Shift in Scholarly Communication Practices? Journal of the American Society for Information Science and Technology, 52(7), 558-569. https://doi.org/10.1002/asi.1097

Cudina, J. N. \& Ossa, J. C. (2016). The top 100 high-impact papers in Colombian psychology: A bibliometric study from WoS and Scopus. Informação \& Sociedade: Estudos, 26(2), 137-154. Recuperado de https://periodicos.ufpb.br/ojs2/index.php/ies/article/view/29200

Cudina, J. N., Millán, J. D. \& Ossa, J. C. (2017). Redes de comunicación científica en la investigación psicológica de las Américas a través de la Revista Interamericana de Psicología. Revista Interamericana de Psicología, 51(3), 283-296. https://doi.org/10.30849/rip/ijp.v51i3.898

De León Sánchez, B. (2011). La relación familia-escuela y su repercusión en la autonomía y responsabilidad de los niños/as. En XXII Congreso Internacional de la Teoría de la Educación. Recuperado de https://extension.uned.es/archivos_publicos/webex_actividades/5385/repercusiones8.pdf

Egido Gálvez, I. (2015). Las relaciones entre familia y escuela. Una visión general. Participación Educativa, 4(7), 11-17.

Epstein, J. L. (2010). School, family, and community partnerships: Preparing educators and improving schools. Westview Press.

Esquivel, J. C., Carbonelli, M. \& Irrazabal, G. (2011). Introducción al conocimiento cientifico y metodología de la investigación social. Florencio Varela: Universidad Nacional Arturo Jauretche.

Fan, X. \& Chen, M. (2001). Parental involvement and students' academic achievement: a meta-analysis, Educational Psychology Review, 13(1), 1-22. https://doi.org/10.1023/A:1009048817385

Faircloth, C. \& R. Rosen (2020). Childhood, parenting culture, and adult-child relations in global perspectives. Families, Relationships and Societies 9(1), 3-6. https://doi.org/10.1332/204674320X15804876175640

Gálvez, C. (2016). Visualización de las principales líneas de investigación en salud pública: un análisis basado en mapas bibliométricos aplicados a la Revista Española de Salud Pública (2006-2015). Revista Española de Salud Pública, 90(1), e1-e10.

Gálvez, I. E. (2020). La colaboración familia-escuela: revisión de una década de literatura empírica en España (2010-2019). Bordón. Revista de Pedagogía 72(3), 65-84. https://doi.org/10.13042/Bordon.2020.79394

García-Castaño, F. J., Rubio-Gómez, M. \& Bouachra, O. (2008). Población inmigrante y escuela en España. Un balance de investigación. Revista de Educación, 345, 23-60.

Garner, R. M., Hirsch, J.A., Albuquerque, F. C. \& Fargen, K. M. (2018). Bibliometric indices: defining academic productivity and citation rates of researchers, departments and journals. Journal of NeuroInterventional Surgery, 10(2), 102-106. https://doi.org/10.1136/neurintsurg-2017-013265

Gómez-García, A., Ramiro, M. T., Ariza, T. \& Granados, M. R. (2012). Estudio bibliométrico de educación XXI. Educación XXI, 15(1), 17-41. Recuperado de http://revistas.uned.es/index.php/educacionXX1/article/view/148 
Gómez-Morales, Y. J. (2015). Usos y abusos de la bibliometría. Revista Colombiana De Antropología, 51(1), $291-307$. https://doi.org/10.22380/2539472X36

Gubbins, V. (2014). Estrategias educativas de familias de clase alta: un estudio exploratorio. Revista Mexicana de Investigación Educativa, 19(63), 1069-1089. Recuperado de https:/www.comie.org.mx/revista/v2018/rmie/ index.php/nrmie/article/view/220

Gubbins, V. (2016). Relación familias y escuelas: ¿por qué y para qué? Santiago, Chile: Ediciones Finis Terrae.

Jafarov, J. (2015). Factors Affecting Parental Involvement in Education: The Analysis of Literature. Khazar Journal of Humanities and Social Sciences, 18(4), 35-44. https://doi.org/10.5782/2223-2621.2015.18.4.35

Jeynes, W. H. (2005). A meta-analysis of the relation of parental involvement to urban elementary school student academic achievement. Urban education 40(3), 237-269. https://doi.org/10.1177/0042085905274540

Jeynes, W. H. (2017). A meta-analysis: The relationship between parental involvement and Latino student outcomes. Education and Urban Society, 49(1), 4-28. https://doi.org/10.1177/0013124516630596

Kock, R., Tulla, A. \& Azevedo, C. (2016). Análisis bibliométrico del concepto de resiliencia aplicado al desarrollo regional. Documents d'Anàlisi Geogràfica, 62(2), 275-298. Recuperado de https://raco.cat/index.php/DocumentsAnalisi/ article/view/308750

Kooij, P. \& Waltman, L., (2019). Vosviewer 1.6. Netherlands: Leiden University Science and Technology Research Center. Recuperado de https://www.vosviewer.com/

Lawani, S. M. (1981). Bibliometrics: Its theoretical foundations, methods and applications. International Journal of Libraries and Information Studies, 31, 294-315. https://doi.org/10.1515/libr.1981.31.1.294

Lotka, A. J. (1926). The frequency distribution of scientific productivity. Journal of the Washington Academy of Sciences, 16, 317-323. https://doi.org/10.1002/asi.4630280610

Madrid, M. J., Jiménez-Fanjul, N., León-Mantero, C. \& Maz-Machado, A. (2017). Revistas brasileńas de Educación en SCOPUS: un análisis bibliométrico. Biblios. Revista de Bibliotecología y Ciencias de la Información, 67, 30-41. https://doi.org/10.5195/biblios.2017.344

Maltseva, D. \& Vladimir, B. (2019). Social network analysis as a field of invasions: bibliographic approach to study SNA development. Scientometrics, 121(6), 1-44. https://oi.org/10.1007/s11192-019-03193-x

Meadows, A. J. (1988). Communication research. San Diego, CA: Academic Press.

Millán, J. D., Polanco, F., Ossa, J. C., Béria, J. S. \& Cudina, J. N. (2017). La cienciometría, su método y su filosofía: Reflexiones epistémicas de sus alcances en el siglo XXI. Revista Guillermo De Ockham, 15(2), 17-27. https://doi.org/10.21500/22563202.3492

Mingers, J. \& Leydesdorff, L. (2015). A review of theory and practice in scientometrics. European Journal of Operational Research, 246(1). 1-19. https://doi.org/10.1016/j.ejor.2015.04.002

Navarro, D., Sánchez, L. \& Gómez, T. (2020). Estudio Bibliométrico de las Publicaciones Científicas sobre la Percepción de las Familias de Alumnos con Necesidades Específicas de Apoyo Educativo. Revista Internacional de Educación para la Justicia Social, 9(1), 231-247. https://doi.org/10.15366/riejs2020.9.1.011

Ortega, M. D. \& Cárcamo, H. (2018). Relación familia-escuela en el contexto rural: miradas desde las familias. Educación 27(52), 81-97. https://doi.org/10.18800/educacion.201801.006

Peña, M., Chávez, P. \& Vergara del Solar, A. (2014). Los niños como agentes políticos: tácticas cotidianas de resistencia en niñas chilenas de estrato socioeconómico medio. Sociedade e cultura 17(2), 291-300. https://doi.org/10.5216/sec.v17i2.29135

Polanco, F., Beria, J. S. \& Klappenbach, H. (2017). Cinco décadas de la Revista Interamericana de Psicología. Un análisis socio-bibliométrico. Revista Interamericana de Psicología/Interamerican Journal of Psychology, 51(3), $297-319$. https://doi.org/10.30849/rip/ijp.v51i3.910

Polanco-Carrasco, R., Gallegos, M., Salas, G. \& López-López, W. (2017). Las revistas de psicología en Chile: historia y situación actual. Terapia Psicológica, 35(1), 81-93. https://doi.org/10.4067/S0718-48082017000100008

Precht, A. (2016). Privatización de la relación entre familias y escuela: un aspecto a considerar en la convivencia escolar. En G. Salas, C. Cornejo, P. Morales \& E. Saavedra (Eds.), Del Pathos al ethos: lineas y perspectivas de la convivencia escolar (pp. 129-142). Talca, Chile: Ediciones UCM. 
Precht, A. (2018). Nos vienen a tirar a sus hijos a la escuela: problematizando la relación entre familia y escuela en tiempos de intensificación de la parentalidad. En D. Ferrada (Ed.), Reflexiones y experiencias educativas desde las comunidades. Investigación en educación para la justicia social (pp. 119-132). Talca, Chile: Ediciones UCM.

Riquelme, S. (2015). Modos de aproximarse al otro en la era del neoliberalismo compensatorio. Expectativas, conversación e intervención posible. Ixtli: Revista Latinoamericana de Filosofía de la Educación, 2(4), 333-353.

Rodríguez-Triana, Z. (2018). Qué y cómo se enseña y aprende en la familia. Un asunto de interés para la escuela. Revista Latinoamericana de Estudios Educativos, 14(2), 132-157. https://doi.org/10.17151/rlee.2018.14.2.7

Ruíz-Marín, L. \& Hernández-Prados, M. Á. (2016). La formación de las familias. Un análisis bibliométrico. Revista Latinoamericana de Estudios de Familia, 8, 9-25. https://doi.org/10.17151/rlef.2016.8.2

Salas, G., Ravelo-Contreras, E., Mejía, S., Andrades, R., Acuña, E., Espinoza, F., .. \& Pérez-Acosta, A. (2018). Dos décadas de Acta Colombiana de Psicología: un análisis bibliométrico. Acta Colombiana de Psicología, 21(2), 13-38. https://doi.org/10.14718/ACP.2018.21.2.2

Salas, G., Vega-Arcea, M., González, C., Ossa, J. C., Cudina, J. N., Caycho-Rodríguez, T., Barboza-Palomino, M., Ventura-León, J., Guerra-Labbé, L. \& López-López, W. (2019). The Fiftieth Anniversary of the Revista Latinoamericana de Psicología: A Bibliometric Analysis. Revista Latinoamericana de Psicología, 51(2), 206-218. https://doi.org/10.14349/rlp.2019.v51.n2.7

Subramanyam, K. (1983). Bibliometric studies of research collaboration: A review. Journal of Information Science, 6(1), 33-38. https://doi.org/10.1177/016555158300600105

Tan, C. Y., Lyu, M. \& Peng, B. (2020) Academic Benefits from Parental Involvement are Stratified by Parental Socioeconomic Status: A Meta-analysis Parenting, 20(4), 241-287, https://doi.org/10.1080/15295192.2019.1694836

Tomás-Górriz, V. \& Tomás-Casterá, V. (2018). La Bibliometría en la evaluación de la actividad científica. Hospital a Domicilio, 2(4), 145-163. https://doi.org/10.22585/hospdomic.v2i4.51

Unesco. (2020). Women in Science. Fact Sheet, 60, 1-4. Recuperado de http://uis.unesco.org/sites/default/files/ documents/fs60-women-in-science-2020-en.pdf

Van Eck, N. J. \& Waltman L. (2010). Software survey: VOSviewer, a computer program for 585 bibliometric mapping. Scientometrics, 84, 523-538. https://doi.org/10.1007/s11192-009-0146-3

Vergara-del Solar, A., Peña, M., Chávez, P. \& Vergara, E. (2015). Los niños como sujetos sociales: El aporte de los Nuevos Estudios Sociales de la infancia y el Análisis Crítico del Discurso. Psicoperspectivas 14(1), 55-65. https://doi.org/10.5027/psicoperspectivas-Vol14-Issue1-fulltext-544

Vergara-del Solar, A., Chávez-Ibarra, P., Peña-Ochoa, M. \& Vergara-Leyton, E. (2016). Experiencias contradictorias y demandantes: la infancia y la adultez en la perspectiva de niños y nińas de Santiago de Chile. Revista Latinoamericana de Ciencias Sociales, Niñez y Juventud, 14(2), 1235-1247. https://doi.org/10.11600/1692715x.14224051115

Vergara-del Solar, A., Sepúlveda, M. \& Salvo, I. (2019). Being a parent and being a child in Chile today: the relational construction of subject positions in a neoliberal context. Subjectivity 12(4), 371-388. https://doi.org/10.1057/s41286-019-00084-7

Vincent, C. (2014). Padres y maestros hacia el diálogo. Una perspectiva inglesa. Profesorado. Revista de Currículum y Formación de Profesorado, 18(2), 35-50. Recuperado de http://hdl.handle.net/20.500.12799/3506 